\title{
Más votos y menos escaños: \\ el impacto del sistema electoral \\ en las elecciones autonómicas \\ catalanas de 2003*
}



RESUMEN

En este artículo revisamos (1) los mecanismos electorales que explican las victorias en escaños, pero no en votos, de CiU en las dos últimas elecciones autonómicas catalanas y (2) las posibles reformas electorales que se pueden acometer. Nuestra principal conclusión es que las mayorías parlamentarias artificiales de CiU no tienen tanto que ver con la desviación del prorrateo como con la variación de las magnitudes de distrito o «efecto de varianza».

Palabras clave: Sistemas Electorales, Cataluña, Desviación del Prorrateo, Efecto de Varianza, Escaños, Votos.

\footnotetext{
* Queremos agradecer los comentarios y observaciones de Agustí Bosch, Joan Botella, Mario Caciagli, Gabriel Colomé, Joan Font, Margarita Gómez-Reino, Jaume Magre, Rafael Martínez, Isidre Molas, Francesc Pallarès y Cesáreo Rodríguez Aguilera, así como la financiación del Ministerio de Ciencia y Tecnología (SEC2000-0758-C02-01) y las facilidades del Centro de Estudios Avanzados en Ciencias Sociales, del Instituto Juan March.
} 
El 16 de noviembre de 2003, en las elecciones celebradas para el Parlamento de Cataluña, el Partit dels Socialistes de Catalunya (PSC) consiguió el mayor número de votos, pero quedó por detrás de Convergència i Unió (CiU) en términos de escaños. Era la segunda ocasión en que el PSC sufría este anómalo resultado. En las elecciones autonómicas catalanas de 1999, el PSC logró 1.183.299 votos (el 37,85 por ciento de los válidos) y CiU unos cinco mil votos menos, 1.178 .420 (el 37,69 por ciento de los válidos). Sin embargo, el PSC hubo de conformarse con 50 escaños, mientras que CiU alcanzó seis más, 56, que le permitieron además formar un gobierno homogéneo minoritario que se mantuvo durante toda la legislatura. En la consulta celebrada en 2003, el PSC (con 1.026.030 votos, o el 31,17 por ciento de los válidos) ganó de nuevo a CiU (con 1.018.115 votos, o el 30,93 por ciento de los válidos). Pero esa diferencia de cerca de ocho mil votos volvió a esfumarse al conseguir el PSC sólo 42 escaños frente a los 46 de $\mathrm{CiU}^{1}$. Y aunque en esta ocasión el descenso de ambos partidos y el crecimiento de Esquerra Republicana de Catalunya (ERC) han posibilitado finalmente un cambio de gobierno, no han impedido, sin embargo, que CiU se atribuyera de nuevo la victoria electoral y aprovechase para endilgar al PSC su permanente condición de partido perdedor.

La doble decepción sufrida por el PSC es comparativamente excepcional, pero no resulta desconocida. En los sistemas electorales mayoritarios, los partidos colocados en segundo lugar por número de votos han terminado disfrutando en algunas ocasiones de mayorías parlamentarias. Así ha ocurrido, por ejemplo, en el Reino Unido o en Canadá; y en Estados Unidos al menos tres candidatos con menos votos que sus competidores se convirtieron en presidentes (Colomer, 2001: cap. 3). En Nueva Zelanda, en 1978 y 1981 el segundo partido en votos llegó incluso a disfrutar de mayorías parlamentarias absolutas, unos resultados que contribuyeron a la sustitución del sistema mayoritario por la variante alemana del sistema proporcional (Mackie y Rose, 1991: 340 ss.; Shugart y Wattenberg, 2001a: 572-573). En los sistemas proporcionales caracterizados por una amplia variabilidad interna de alguno de sus elementos y/o una distribución territorial del voto asimétrica, los resultados consistentes en más votos y menos escaños son asimismo posibles ${ }^{2}$. Un caso ilustrativo es el del sistema electoral del Congreso de los Diputados, caracterizado precisamente por la acusada diferencia en el número de escaños que se eligen en sus circunscripciones. En combinación con otros mecanismos como la desviación del prorrateo, esta heterogeneidad institucional y política determina un premio de localización del voto: cuanto más se concentren los votos de un partido en las circunscripciones más pequeñas (o mayor sea la correlación negativa entre la

\footnotetext{
1 Los datos electorales de 1999 proceden de Linz, Montero y Ruiz (2004), www.lavanguardia.es y www.parlament-cat.net, y los de 2003 están obtenidos de La Vanguardia y El País, ambos de 17 de noviembre de 2003.

2 Una variante radical de esta situación son las «minorías artificiales», como las denomina Rae (1971: 74), consistentes en partidos con mayorías electorales absolutas que terminan obteniendo una pluralidad o mayoría relativa de escaños; cf. también Lijphart (1995: 120-121).
} 
distribución de su voto y la magnitud de los distritos), mayor es su sobrerrepresentación en escaños, y a la inversa (cf. Penadés, 1999). En las elecciones de 1996, por ejemplo, los resultados electorales agregados arrojaron un virtual empate entre el Partido Popular (PP) y el Partido Socialista Obrero Español (PSOE): el primero superó al segundo por algo menos de trescientos mil votos. No obstante, la victoria del PP en los distritos más pequeños (con entre uno y cinco escaños), y en consecuencia los más rentables en términos de escaños, frente a la del PSOE en los más grandes (con entre 10 y 34), y menos rentables, facilitó la conversión del escaso punto porcentual de diferencia en votos en más de cuatro puntos en escaños (cf. Lago, 2003: cap. 7). Quiere esto decir que si la distribución territorial de los apoyos electorales hubiera sido la inversa, el PSOE habría ganado las elecciones en escaños, pero no en votos. En el ámbito autonómico, las elecciones para el Parlamento del País Vasco en 1986 proporcionan otro caso de interés. Debido a la extraordinaria desviación del prorrateo en las tres circunscripciones y a la mayor presencia de los partidos nacionales en Álava y de los nacionalistas en Vizcaya, el Partido Socialista de Euskadi-Euskadiko Esquerra (PSEPSOE) consiguió 19 escaños con el 22 por ciento de los votos válidos, mientras que el Partido Nacionalista Vasco (PNV) obtuvo sólo 17 con el 23,6 por ciento.

Los sistemas electorales suelen ser fáciles víctimas propiciatorias de cuantos defectos se les adjudican para dar cuenta de resultados electorales imprevistos o sorprendentes. En las elecciones para el Congreso de los Diputados, por ejemplo, las supuestas vinculaciones de la «ley D'Hondt» con la magnitud de los distritos, la desproporcionalidad o la naturaleza de las listas nutren periódicamente unos comentarios poselectorales que, por lo general, acaban solicitando la necesaria reforma del sistema electoral (Montero, 1997). En Cataluña, la inexistencia de una ley electoral propia ha impedido convertirla en responsable de las anomalías sufridas por el PSC ${ }^{3}$. Pero el impacto de su principal componente distintivo, radicado en la peculiar asignación de escaños a las cuatro circunscripciones catalanas, ha sido considerado especialmente grave. Desde 1984, la diferente magnitud de las circunscripciones ha generado una cierta desproporcionalidad que, a su vez, ha ocasionado la aparición de mayorías «manufacturadas» (o artificiales): CiU ha visto así transformar su pluralidad (o mayoría relativa) de votos en mayorías absolutas parlamentarias. No obstante, su intervención en las dos últimas elecciones se ha producido aparentemente en otra dirección y, en todo caso, ha resultado ser mucho más onerosa. Se habría pasado así de corregir al alza en escaños las victorias de un partido

\footnotetext{
3 Pese a su considerable actividad legislativa y a la amplitud de su autogobierno, cabe recordar que Cataluña es la única Comunidad Autónoma que carece todavía de una ley electoral propia, pese a los más de veinte años transcurridos desde la celebración de sus primeras elecciones autonómicas. En la Disposición Transitoria $4 .^{a}$ del Estatuto de Autonomía de Cataluña de 1979 se establecen unas normas elementales sobre convocatoria electoral, magnitud de las circunscripciones, características del sufragio, ámbito de las Juntas electorales, sistema de recursos y aplicación subsidiaria de la legislación prevista para las elecciones al Congreso de los Diputados «en tanto una Ley de Cataluña no regule el procedimiento para las elecciones al Parlamento». Poco antes de la finalización de la primera legislatura catalana, el Parlamento aprobó la Ley 5/1984; su único artículo señalaba la vigencia del Decreto-ley de marzo de 1977, que fue luego obviamente sustituido por la Ley Orgánica del Régimen Electoral General (LOREG), de mayo de 1985. Cf. Vallès (1990).
} 
en votos a determinar qué partido gana en escaños, y por lo tanto gobierna, sin que parezca importar cuál gana en votos. Dicho todavía de otra forma, si hasta ahora el partido que ganaba las elecciones con una pluralidad de votos conseguía además una mayoría absoluta de escaños, en las elecciones de 1999 y 2003 el partido con más votos (el PSC) ha perdido las elecciones al recibir menos escaños que su inmediato competidor (CiU).

¿Puede un componente del sistema electoral producir una consecuencia y su contraria? Así han parecido entenderlo en Cataluña las elites partidistas, los medios de comunicación, los observadores políticos y los expertos académicos, que han criticado las desviaciones en el prorrateo como el elemento responsable de los resultados dispares en votos y escaños y han coincidido también en la necesidad de que el Parlamento apruebe de una vez una ley electoral catalana que las impida. En este artículo queremos discutir ambos supuestos. Analizaremos, en primer lugar, los mecanismos a través de los cuales la agregación de los resultados de las diversas circunscripciones catalanas permite que un partido que no haya sido vencedor en términos de votos obtenga, sin embargo, al menos una pluralidad de los escaños. $Y$ examinaremos después distintas alternativas que pueden evitar o minimizar este resultado irregular, incluyendo algunas de las propuestas realizadas por los partidos o por expertos e incluidas dentro de proyectos más o menos acabados de un sistema electoral propio para Cataluña. Vaya por delante nuestra opinión de que las desviaciones del prorrateo, que han sido tenidas de forma casi unánime como las responsables únicas del resultado de más votos y menos escaños, son relevantes a este respecto, pero no son las únicas que cuentan. En las páginas que siguen intentaremos demostrar que las victorias en escaños de CiU con una minoría de votos tienen que ver fundamentalmente con el llamado «efecto de varianza» de las magnitudes de las circunscripciones (Monroe y Rose, 2002), de modo que las eventuales correcciones a aquellas desviaciones pueden aparecer sólo como soluciones provisionales e inciertas a un problema más estructural. Los resultados de este estudio deben, pues, entenderse como un paso necesario para explicar el funcionamiento del sistema electoral catalán y sopesar el alcance de sus posibles reformas.

\section{LOS COMPONENTES DEL SISTEMA ELECTORAL CATALÁN}

¿Cómo ha funcionado el sistema electoral catalán? En esta sección examinaremos sus principales componentes, los compararemos con los de otras Comunidades Autónomas y destacaremos algunas de sus características. Esta revisión nos permitirá luego discutir con mayor detenimiento los factores que puedan explicar el peculiar impacto de las reglas electorales en las dos últimas consultas autonómicas, cuyos resultados se recogen en la tabla 1. Como ya se ha apuntado, la inexistencia de una ley electoral propia, pese a los más de veinte años de autogobierno transcurridos y a las siete consultas autonómicas celebradas, cifra la regulación catala- 
na en la Disposición Transitoria 4. ${ }^{a}$ del Estatuto de 1979. Allí se establece que el Parlamento estará integrado por 135 diputados y que las circunscripciones serán las cuatro provincias. Y en el artículo 31 se añade que el Parlamento será elegido «por un término de cuatro años, por sufragio universal, libre, igual, directo y secreto (...). El sistema electoral será de representación proporcional y asegurará, además, la adecuada representación de todas las zonas del territorio de Cataluña». De acuerdo con las previsiones de la Ley catalana 5/1984, en todo lo demás rige la Ley Orgánica del Régimen Electoral General (LOREG), de mayo de 1985.

TABLA 1

Resultados de las elecciones autonómicas catalanas de 1999 y 2003 (a)

19 de octubre de 1999

\begin{tabular}{|c|c|c|c|c|c|c|c|c|c|c|}
\hline \multirow{3}{*}{$\frac{\text { Partidos }}{\text { IC-V (b) }}$} & \\
\hline & \multicolumn{3}{|c|}{ Barcelona } & \multicolumn{2}{|l|}{ Girona } & Lleida & \multicolumn{2}{|c|}{ Tarragona } & \multicolumn{2}{|c|}{ Cataluña } \\
\hline & 3,30 & 3 & - & - & - & - & - & - & 2,51 & 3 \\
\hline ERC & 7,71 & 7 & 12,76 & 2 & 15,57 & 1 & 10,46 & 2 & 8,65 & 12 \\
\hline PSC-CpC (c) & 39,91 & 36 & - & - & - & - & - & - & 30,25 & 36 \\
\hline PSC-CpC-IC-V (d) & - & & 29,12 & 5 & 29,41 & 5 & 34,00 & 6 & 7,50 & 16 \\
\hline $\mathrm{CiU}$ & 35,07 & 31 & 48,38 & 9 & 47,92 & 8 & 41,16 & 8 & 37,6 & 56 \\
\hline PP & 9,89 & 8 & 6,03 & 1 & 7,95 & 1 & 10,59 & 2 & 9,50 & 12 \\
\hline Otros & 4,12 & - & 3,72 & - & 3,16 & - & 3,79 & - & 3,99 & 一 \\
\hline \multirow[t]{2}{*}{ Total } & 100,00 & 85 & 100,00 & 17 & 100,00 & 15 & 100,00 & 18 & 100,00 & 135 \\
\hline & \multicolumn{10}{|c|}{16 de noviembre de 2003} \\
\hline Partidos & \multicolumn{2}{|c|}{ Barcelona } & \multicolumn{2}{|r|}{ Girona } & & Lleida & \multicolumn{2}{|c|}{ Tarragona } & \multicolumn{2}{|c|}{ Cataluña } \\
\hline ICV-EUiA (b) & 8,06 & 7 & 5,33 & 1 & 4,34 & - & 5,18 & 1 & 7,30 & 9 \\
\hline $\mathrm{ERC}$ & 15,18 & 13 & 21,95 & 4 & 19,91 & 3 & 19,05 & 3 & 16,47 & 23 \\
\hline PSC-CpC (c) & 33,18 & 29 & 23,64 & 4 & 22,43 & 4 & 28,22 & 5 & 31,17 & 42 \\
\hline CiU & 28,75 & 25 & 38,70 & 7 & 41,50 & 7 & 33,77 & 7 & 30,93 & 46 \\
\hline PP & 12,53 & 11 & 8,04 & 1 & 9,65 & 1 & 11,74 & 2 & 11,87 & 15 \\
\hline Otros & 2,30 & - & 2,34 & - & 2,17 & - & 3,04 & - & 2,26 & - \\
\hline Total & 100,00 & 85 & 100,00 & 17 & 100,00 & 15 & 100,00 & 18 & 100,00 & 135 \\
\hline
\end{tabular}

(a) En cada distrito, la primera columna recoge las proporciones de voto válido de cada partido; y la segunda, el número de escaños conseguidos.

(b) En 1999, Iniciativa per Catalunya (IC) se coligó con Verds (V), y ambos a su vez con el PSC, en las circunscripciones de Girona, Lleida y Tarragona; en 2003, fusionados con Verds y en coalición con Esquerra Unida i Alternativa (EUiA).

(c) En coalición con Ciutadans pel Canvi (CpC) en Barcelona en 1999 y en las cuatro circunscripciones en 2003.

(d) En 1999, esta coalición se presentó en las circunscripciones de Girona, Lleida y Tarragona.

FUENTES:

Linz, Montero y Ruiz (2004); www.lavanguardia.es; www.parlament-cat.net, La Vanguardia y El País, 17 de noviembre de 2003. 


\section{El prorrateo electoral}

La Disposición Transitoria 4. ${ }^{a}$ del Estatuto establece asimismo que la circunscripción de Barcelona elige un escaño por cada 50.000 habitantes, con un máximo de 85, y que las de Girona, Lleida y Tarragona eligen un mínimo de 6 escaños, más uno por cada 40.000 habitantes, atribuyéndoles 17, 15 y 18 diputados, respectivamente. Como comprobaremos en seguida, la distribución de los escaños entre las provincias tiene unas obvias consecuencias inmediatas sobre la magnitud de las circunscripciones. Pero tiene implicaciones aún más relevantes sobre el valor del voto de los electores integrados en cada una de ellas. La mecánica del prorrateo electoral, basada en un doble criterio territorial y poblacional, produce unos desequilibrios muy intensos en la influencia individual de los votos. El mínimo inicial de seis diputados con los que cuentan Girona, Lleida y Tarragona supone que 18 escaños, es decir, más del 13 por ciento del total, se reparten discriminando al máximo a Barcelona. La distribución de los 117 escaños restantes según dos criterios poblacionales distintos para Barcelona y el resto de los distritos acentúa el prorrateo desviado ${ }^{4}$. Además, estos desequilibrios se han agravado con el paso del tiempo. La aplicación del prorrateo inicial se hizo con los datos censales correspondientes a 1976. Después, el progresivo crecimiento del censo electoral ha afectado las ratios del prorrateo en todas las circunscripciones, pero, como cabía esperar, su incidencia ha sido especialmente fuerte en Barcelona ${ }^{5}$. En consecuencia, las provincias menos pobladas y más rurales o, en otras palabras, las que distribuyen menos escaños, se encuentran notablemente sobrerrepresentadas, mientras que la más poblada o urbana sufre una aguda infrarrepresentación ${ }^{6}$. Los distritos extremos que habitualmente sirven como referencia son Lleida, donde, de acuerdo con el censo electoral de 2003, bastan 20.150 electores para elegir un diputado, y Barcelona, donde se necesitan 46.291. En otras palabras, el voto de un ciudadano de Lleida tiene, en términos de representación parlamentaria, un valor más del doble superior al de uno en Barcelona.

En una perspectiva comparada en España, la desviación del prorrateo en el sistema electoral catalán, calculada de acuerdo con el índice de desproporcionalidad de Loosemore y

${ }^{4}$ Conocido también como malapportionment, el prorrateo desviado designa la discrepancia entre los porcentajes de representación legislativa en términos de escaños y los del censo electoral en cada una de las circunscripciones; cf. Gallagher (1991) y Monroe (1994).

5 Entre 1980 y 2003, el censo electoral catalán ha crecido en alrededor de 915.000 personas, de las cuales unas 530.000 corresponden a Barcelona, unas 125.000 a Girona y a Tarragona, y unas 31.000 a Lleida; eso significa que el 58 por ciento de las variaciones del censo de Cataluña radica exclusivamente en el crecimiento del de Barcelona. Cf. también Pallarès (1998: 231).

6 Aunque los términos urbano y rural son poco precisos y resultan relativamente inapropiados, acudiremos a ellos por su utilización convencional en la caracterización diferenciada de las cuatro circunscripciones catalanas. Por lo demás, ese uso se ajusta a la tendencia general que parece vincular el malapportionment con los distritos rurales y políticamente conservadores a expensas de los urbanos y más políticamente progresistas; cf. Samuels y Snyder (2001: 668) y Monroe y Rose (2002: 69). 
Hanby $(1971)^{7}$, se encuentra cerca de la media y la mediana de la distribución (tabla 2). Canarias, Baleares, País Vasco, Aragón, Comunidad Valenciana y Galicia, en este orden, tienen un malapportionment mayor. El índice español para el Congreso de los Diputados es bastante similar al catalán, como se evidencia en el ya conocido contraste entre los 26.508 sorianos existentes por escaño, según el censo electoral de 2000, y los 130.097 barceloneses o los 126.974 madrileños. En los 70 sistemas electorales de 27 países democráticos analizados por Lijphart (1995: 183), el español (y, a fortiori, el catalán) está entre los nueve casos con mayores niveles de sobrerrepresentación rural. Y en la muestra de 78 Parlamentos democráticos seleccionados por Samuels y Snyder (2001), el índice de malapportionment catalán estaría situado entre los 15 países con mayores desviaciones de prorrateo, justo por encima del de España, sólo superados por Islandia entre los europeos y encabezados por distintos países africanos y latinoamericanos.

TABLA 2

Índices de desviación del prorrateo en los sistemas electorales autonómicos y en el del Congreso de los Diputados en las últimas elecciones celebradas en cada caso (a)

\begin{tabular}{|c|c|c|c|c|c|}
\hline Puesto & $\begin{array}{r}\text { Comunidades } \\
\text { Autónomas }\end{array}$ & $\begin{array}{r}\text { Índice de } \\
\text { desviación (b) }\end{array}$ & Puesto & $\begin{array}{r}\text { Comunidades } \\
\text { Autónomas }\end{array}$ & $\begin{array}{r}\text { Índice de } \\
\text { desviación (b) }\end{array}$ \\
\hline 1 & Canarias & 0,3485 & 10 & España & 0,0963 \\
\hline 2 & Baleares & 0,2483 & 11 & Extremadura & 0,0822 \\
\hline 3 & País Vasco & 0,2079 & 12 & Castilla y León & 0,0821 \\
\hline 4 & Aragón & 0,1933 & 13 & Asturias & 0,0572 \\
\hline 5 & Comunidad Valenciana & 0,1420 & 14 & Murcia & 0,0440 \\
\hline 6 & Galicia & 0,1285 & 15 & Cantabria & 0,00 \\
\hline 7 & Cataluña & 0,1233 & 16 & Madrid & 0,00 \\
\hline 8 & Andalucía & 0,1119 & 17 & Navarra & 0,00 \\
\hline 9 & Castilla-La Mancha & 0,1115 & 18 & La Rioja & 0,00 \\
\hline
\end{tabular}

(a) Las elecciones al Congreso de los Diputados y autonómicas de Andalucía se celebraron en marzo de 2000; las autonómicas de País Vasco y Galicia, en mayo y octubre de 2001, respectivamente; las de Cataluña, en noviembre de 2003; y las de las restantes Comunidades, en mayo de 2003.

(b) La media de la desviación del prorrateo para todas las Comunidades Autónomas es 0,1098; la moda, 0; y la desviación típica, 0,0944 .

FUENTE:

Elaboración propia.

7 El índice se computa a partir de la siguiente fórmula: $M A L$ [por malapportionment] $=(1 / 2) \sum\left|s_{i}-v_{i}\right|$, donde sigma se refiere a la suma de todos los distritos $i, s_{i}$ es el porcentaje de escaños asignados al distrito $i$, y $v_{i}$ es el porcentaje de la población que reside en el distrito $i$. Un índice de, por ejemplo, 0,12 significa que el 12 por ciento de los escaños de un determinado Parlamento está distribuido de forma que viola el principio de «una persona, un voto»; cf. Loosemore y Hanby (1971). 


\section{La magnitud de las circunscripciones}

El reparto de los 135 diputados entre las cuatro circunscripciones catalanas supone que su magnitud media es 33,75 escaños y la mediana 17,5; el umbral efectivo coincide con el 3 por ciento de la barrera legal ${ }^{8}$. Se trata, por supuesto, de una magnitud elevada. Entre los sistemas electorales autonómicos, el catalán es el que dispone del mayor número de escaños y el que tiene las circunscripciones más grandes ${ }^{9}$ (tabla 3 ). Sin embargo, la utilidad de las medidas de tendencia central para caracterizar la permisividad del sistema electoral catalán es limitada: la desviación típica de la distribución de circunscripciones $(34,19)$, la mayor en perspectiva comparada, es más alta que la media. Su rango oscila así entre los 15 escaños de Lleida y los 85 de Barcelona, pasando por los 17 de Girona y los 18 de Tarragona. Si los efectos de los sistemas electorales dependen fundamentalmente de la magnitud de sus distritos, las circunscripciones catalanas garantizan una elevada proporcionalidad, sobre todo en Barcelona. Pese a ello, y como veremos más adelante, las diferencias en las magnitudes de las circunscripciones catalanas tienen un efecto significativo sobre la proporcionalidad y sobre las primas obtenidas por los partidos mayoritarios. Esta acusada permisividad electoral se acompaña, por supuesto, de una elevada fragmentación del sistema de partidos: Cataluña cuenta con la mayor oferta partidista en España, tanto en su versión electoral como parlamentaria, de acuerdo con los índices del número efectivo de partidos propuestos por Laakso y Taagepera (1979) ${ }^{10}$ (tabla 4).

${ }^{8}$ El umbral efectivo en una circunscripción es el porcentaje de votos que posibilita conseguir algún escaño; cf. Lijphart (1995: 63).

9 Al parecer, la cifra de 135 escaños fue adoptada por los redactores del Estatuto catalán para igualar en el futuro Parlamento los resultados obtenidos por los partidos en las elecciones legislativas de 1977 y 1979; cf. Colomé (1998: 296).

10 El número efectivo de partidos electorales se computa a partir de la siguiente fórmula: $N E P E=1 / \Sigma v_{i}^{2}$, donde sigma se refiere a la suma de todos los partidos $i$, y $v_{i}$ es la proporción de votos del partido $i$. En el cálculo del número efectivo de partidos parlamentarios simplemente se sustituye la proporción de votos por la de escaños, $p_{i}: N E P P=1 / \sum p_{i}^{2}$; cf. Laakso y Taagepera (1979). 
TABLA 3

Magnitudes de las circunscripciones en los sistemas electorales autonómicos y en el Congreso de los Diputados en las últimas elecciones celebradas en cada caso*

\begin{tabular}{lrrrr} 
Comunidades Autónomas & Escaños & Circunscripciones & Magnitud media & Desviación típica \\
\hline Andalucía & 109 & 8 & 13,63 & 2,67 \\
Aragón & 67 & 3 & 22,33 & 11,15 \\
Asturias & 45 & 3 & 15 & 15,62 \\
Baleares & 59 & 4 & 14,75 & 13,32 \\
Canarias & 60 & 7 & 8,57 & 4,79 \\
Cantabria & 39 & 1 & 39 & 0 \\
Castilla-La Mancha & 47 & 5 & 9,4 & 1,82 \\
Castilla y León & 83 & 9 & 9,22 & 3,38 \\
Cataluña & 135 & 4 & 33,75 & 34,19 \\
Extremadura & 65 & 2 & 32,50 & 3,54 \\
Galicia & 75 & 4 & 18,75 & 4,99 \\
Madrid & 111 & 1 & 111 & 0 \\
Murcia & 45 & 5 & 9 & 7,25 \\
Navarra & 50 & 1 & 50 & 0 \\
La Rioja & 33 & 1 & 33 & 0 \\
País Vasco & 75 & 3 & 25 & 0 \\
Comunidad Valenciana & 89 & 3 & 29,67 & 6,51 \\
España & & & & 5,93 \\
Media & 350 & 52 & & 6,40
\end{tabular}

* Las elecciones al Congreso de los Diputados y autonómicas de Andalucía se celebraron en marzo de 2000; las autonómicas de País Vasco y Galicia, en mayo y octubre de 2001, respectivamente; las de Cataluña, en noviembre de 2003; y las de las restantes Comunidades, en mayo de 2003.

FUENTE:

Elaboración propia. 
TABLA 4

Índices de fragmentación electoral y parlamentaria en los sistemas electorales autonómicos y del Congreso de los Diputados en las últimas elecciones celebradas en cada caso (a)

\begin{tabular}{lccr} 
Comunidades Autónomas & $\begin{array}{c}\text { Número efectivo } \\
\text { de partidos electorales }\end{array}$ & $\begin{array}{c}\text { Número efectivo } \\
\text { de partidos parlamentarios }\end{array}$ & Diferencia \\
\hline Cataluña & 4,10 & 3,87 & 0,23 \\
Navarra & 3,93 & 3,50 & 0,43 \\
Canarias & 3,62 & 3,23 & 0,39 \\
País Vasco & 3,55 & 3,35 & 0,20 \\
Aragón & 3,54 & 3,30 & 0,24 \\
Baleares & 3,50 & 3,11 & 0,39 \\
Cantabria & 3,10 & 2,73 & 0,37 \\
Asturias & 2,89 & 2,35 & 0,54 \\
Andalucía & 2,76 & 2,43 & 0,33 \\
Comunidad Valenciana & 2,70 & 2,22 & 0,48 \\
Galicia & 2,65 & 2,49 & 0,16 \\
Castilla y León & 2,55 & 2,05 & 0,50 \\
Madrid (b) & 2,45 & 2,30 & 0,15 \\
La Rioja & 2,48 & 2,23 & 0,25 \\
Extremadura & 2,31 & 2,13 & 0,18 \\
Murcia & 2,20 & 1,95 & 0,25 \\
Castilla-La Mancha & 2,07 & 1,90 & 0,27 \\
España & & 2,48 & 0,54 \\
Media & 3,02 & 2,64 & 0,33
\end{tabular}

(a) Las elecciones al Congreso de los Diputados y autonómicas de Andalucía se celebraron en marzo de 2000; las autonómicas de País Vasco y Galicia, en mayo y octubre de 2001, respectivamente; las de Cataluña, en noviembre de 2003; y las de las restantes Comunidades (excepto Madrid), en mayo de 2003.

(b) Según los resultados de las segundas elecciones autonómicas, celebradas el 26 de octubre de 2003.

FUENTE:

Elaboración propia.

\section{La fórmula electoral y la barrera legal}

Como es sabido, la fórmula D’Hondt se basa en la minimización de la mayor desviación relativa de la proporcionalidad en la asignación a cada partido. Con más precisión, este método busca un divisor tal que la suma de los números enteros que resultan de dividir así los votos de los partidos sea igual al número de escaños. Los escaños se conceden a los partidos en número igual a dichos cocientes enteros, de manera que a cada partido le corresponden 
$E$ escaños si, para algún valor de $X$, los cocientes de los votos por el divisor se encuentran en el intervalo $E_{i} \leq V_{i} / X \leq E_{i}+1$ (Penadés, 2000: cap. 4). Y aunque la fórmula D’Hondt tiene una igualmente bien conocida tendencia a favorecer a los partidos grandes en cada distrito, esa tendencia no depende de la barrera de entrada para el primer escaño (que, de hecho, es la misma que la cuota Droop: 1 / [M + 1]), sino de las sucesivas (Penadés, 1999: 297). En cualquier caso, este efecto de la fórmula está en función de nuevo de la magnitud de la circunscripción en la que se aplique. En distritos tan grandes como los cuatro del sistema electoral catalán, no afecta apenas a la proporcionalidad electoral.

Tanto la fórmula D'Hondt como la barrera legal del 3 por ciento de los votos válidos emitidos en la circunscripción proceden del sistema electoral español para el Congreso de los Diputados y se han incorporado subsidiariamente en el sistema electoral catalán. Y si bien en las elecciones generales se trata de dos elementos explícitamente introducidos para reducir la proporcionalidad, en las catalanas apenas cuentan en la medida en que interaccionan con magnitudes de circunscripción elevadas. Así, la superación de la barrera legal garantiza la consecución de algún escaño sólo en Barcelona. Para que en los otros tres distritos este 3 por ciento de los votos supere el umbral de inclusión ${ }^{11}$ es necesario que el número de partidos en competición sea tan alto como impensable. Si aceptamos, siguiendo a Taagepera y Shugart (1989) y Lijphart (1995: 64), que el número de competidores en cada distrito sea el mismo que su magnitud y que el umbral efectivo se sitúe a medio camino entre los umbrales de inclusión y de exclusión, sólo puede conseguirse algún escaño con el 3 por ciento de los votos en circunscripciones con 25 o más escaños, esto es, sólo en Barcelona ${ }^{12}$.

\section{Los rendimientos del sistema electoral y los perfiles de proporcionalidad}

Pese a que los resultados de las dos últimas elecciones autonómicas parezcan indicar lo contrario, la proporcionalidad del sistema electoral catalán es elevada. De acuerdo con el índice de Rose $^{13}$, la proporcionalidad se aproxima al 97 por ciento en las elecciones de 2003,

\footnotetext{
11 El umbral de inclusión o de representación es el porcentaje mínimo de votos con el que un partido puede obtener un escaño en las condiciones más favorables posibles; el de exclusión, el porcentaje máximo de votos que, en las condiciones más desfavorables posibles, puede resultarle insuficiente para obtener un escaño; cf. Lijphart (1995: 63-64). 12 El umbral efectivo se ha calculado de acuerdo con la fórmula siguiente: $U_{e f}=\frac{50 \%}{(M+1)}+\frac{50 \%}{2 M}$, donde $M$ es la magnitud de la
circunscripción; cf. Lijphart (1995: 66).

${ }^{13}$ El índice de Rose se calcula sumando las diferencias absolutas entre los porcentajes de voto y escaños de cada partido, dividiéndolas entre dos y restándolas de cien, de acuerdo con la siguiente fórmula: $P=100-1 / 2 \sum\left|v_{i}-e_{j}\right|$, donde $v$ y e son el porcentaje de votos y escaños del partido $i$. El índice oscila entre 0 (mínima proporcionalidad) y 100 (máxima proporcionalidad); cf. Rose (1984).
} 
mientras que, según el índice de Gallagher ${ }^{14}$, la desproporcionalidad supera ligeramente los 2 puntos. De este modo, el sistema electoral catalán es el tercero más proporcional, o el cuarto menos desproporcional, de los que se emplean en España (tabla 5). La consecuencia partidista más inmediata de esta proximidad entre los porcentajes de votos y los de escaños de los distintos competidores es que las primas electorales que consiguen los partidos mayoritarios no son demasiado importantes, sobre todo si las comparamos con las que se obtienen, por ejemplo, en las elecciones al Congreso de los Diputados (Lago, 2003: 194197). Además, aumentan en principio los partidos viables ${ }^{15}$ al necesitar menos votos para

TABLA 5

Índices de proporcionalidad (Rose) y de desproporcionalidad (Gallagher) en los sistemas electorales autonómicos y en el del Congreso de los Diputados en las últimas elecciones celebradas en cada caso (a)

\begin{tabular}{|c|c|c|c|c|c|c|c|}
\hline Puesto (b) & $\begin{array}{r}\text { Comunidades } \\
\text { Autónomas }\end{array}$ & $\begin{array}{l}\text { Índice de } \\
\text { Rose (c) }\end{array}$ & $\begin{array}{r}\text { Índice de } \\
\text { Gallagher (d) }\end{array}$ & Puesto (b) & $\begin{array}{r}\text { Comunidades } \\
\text { Autónomas }\end{array}$ & $\begin{array}{l}\text { Índice de } \\
\text { Rose (c) }\end{array}$ & $\begin{array}{r}\text { Índice de } \\
\text { Gallagher (d) }\end{array}$ \\
\hline 1 & Galicia & 97,39 & 1,73 & 10 & Murcia & 94,44 & 4,30 \\
\hline 2 & Madrid (e) & 97,14 & 1,69 & 11 & Cantabria & 93,67 & 4,06 \\
\hline 3 & Cataluña & 96,73 & 2,24 & 12 & Andalucía & 93,51 & 4,33 \\
\hline 4 & País Vasco & 96,51 & 2,24 & 13 & Canarias & 92,31 & 4,76 \\
\hline 5 & Aragón & 96,48 & 2,03 & 14 & España & 91,43 & 5,61 \\
\hline 6 & Extremadura & 96,22 & 2,85 & 15 & Castilla y León & 90,98 & 6,82 \\
\hline 7 & Castilla-La Mancha & 95,77 & 3,02 & 16 & Com. Valenciana & 90,88 & 6,18 \\
\hline 8 & Navarra & 95,50 & 3,26 & 17 & Asturias & 90,41 & 6,31 \\
\hline 9 & La Rioja & 94,52 & 3,70 & 18 & Baleares & 89,80 & 5,14 \\
\hline
\end{tabular}

(a) Las elecciones al Congreso de los Diputados y autonómicas de Andalucía se celebraron en marzo de 2000; las autonómicas de País Vasco y Galicia, en mayo y octubre de 2001, respectivamente; las de Cataluña, en noviembre de 2003; y las de las restantes Comunidades (excepto Madrid), en mayo de 2003.
(b) b Según el índice de Rose.
(c) La media de proporcionalidad para todas las Comunidades es 94,09; la desviación típica, 2,56.
(d) La media de desproporcionalidad para todas las Comunidades es 3,90; la desviación típica, 1,65.
(e) Según los resultados de las segundas elecciones autonómicas, celebradas el 26 de octubre de 2003.

FUENTE:

Elaboración propia.

14 El índice de desproporcionalidad de Gallagher se calcula sumando las diferencias al cuadrado entre los porcentajes de votos y escaños de cada partido, dividiéndolas entre dos y sacando las raíz cuadrada, de acuerdo con la siguiente fórmula:

$M C=\sqrt{\frac{1}{2} \sum(v i-e l)^{2}}$, donde $v$ y e son el porcentaje de votos y escaños del partido $i$. El índice oscila entre 0 (mínima desproporcionalidad) y 100 (máxima desproporcionalidad); cf. Gallagher (1991) y, más generalmente, Taagepera y Grofman (2003).

15 Un partido o candidato es viable cuando, de acuerdo con sus expectativas electorales, espera conseguir algún escaño. 
obtener algún escaño, lo que de paso contribuye a generar una escasa cantidad de votos sin representación. De todos modos, cabe imaginar que este resultado agregado esconde dinámicas distintas en las cuatro circunscripciones catalanas. Como no podía ser de otra manera, la extraordinaria variabilidad en la magnitud de los distritos supone que los niveles de proporcionalidad electoral presentan acusadas diferencias. En efecto, si en la provincia de Barcelona la proporcionalidad se aproxima al 99 por ciento, en la de Lleida, por ejemplo, apenas supera el 91 por ciento. En otras palabras, la proporcionalidad en Barcelona es 7,5, un 3,7 y un 2,7 por ciento mayor que en Lleida, Tarragona y Girona, respectivamente (véase infra, tabla 7).

Podemos observar con mayor facilidad la relación existente entre la magnitud de las circunscripciones y las primas que los partidos consiguen en cada una de ellas y en Cataluña mediante sus respectivos perfiles de proporcionalidad (Taagepera y Shugart, 1989: cap. 7) ${ }^{16}$. Estos perfiles nos muestran las distintas tasas de ventaja de los partidos en función de su porcentaje de $\operatorname{votos}^{17}$. Si un partido carece de representación, la tasa de ventaja sería $A=0$. Si consigue escaños, pero proporcionalmente menos de los que corresponderían de acuerdo con sus votos, $A$ estaría entre 0 y 1.Y si un partido alcanza más escaños de los que le corresponderían proporcionalmente de acuerdo con sus votos, $A$ estaría por encima de 1 . E punto $A=1$ y la hipotética línea que arranca de él para cortar el gráfico paralelamente al eje de abcisas representan la proporcionalidad perfecta, mientras que la línea curva reflejaría la pauta media de las ventajas y desventajas de los partidos ${ }^{18}$. La trayectoria de la línea del perfil nos permite comprobar qué tipo de ventaja o desventaja puede esperar un partido según su volumen de voto. En general, los perfiles se clasifican en cuatro categorías puras según el punto de corte (o punto $B$ ) entre el perfil y la línea hipotética de proporcionalidad: planos ( $B$ es menor del 5 por ciento), de elevación adelantada ( $B$ se encuentra entre el 5 y el 15 por ciento), de elevación atrasada ( $B$ se encuentra entre el 15 y el 25 por ciento) y de elevación muy atrasada ( $B$ es mayor que el 25 por ciento). De este modo, el punto $B$ determina la neutralidad del sistema electoral, es decir, a partir de qué porcentaje de voto un partido no

\footnotetext{
16 Para contar con un número razonable de observaciones hemos utilizado los resultados electorales de 1999 y de 2003 . Para análisis similares del sistema electoral para el Congreso de los Diputados pueden verse Montero, Llera y Torcal (1992) y Penadés (1999)

17 La tasa de ventaja, o advantage ratio (Taagepera y Shugart, 1989: 68), se obtiene mediante la sencilla fórmula de $A=\%$ escaños / \% votos.

18 Para trazar los perfiles de proporcionalidad hemos empleado la técnica conocida como Loess o Louvess. Su realización exige, en pocas palabras, que para cada dato de una muestra se ajuste una regresión polinomial localmente ponderada. Es una regresión local porque sólo se emplea el subconjunto de observaciones que están en la cercanía del punto que se ajusta en el modelo de regresión; y es ponderada en el sentido de que se otorga menos peso a las observaciones que están más lejos del punto dado. La curva sigue la tendencia central de los valores de la variable dependiente a lo largo del rango de la variable independiente sin que se especifique la forma funcional de la relación. Puesto que el método de los mínimos cuadrados es muy sensible a la presencia de observaciones atípicas, se ha optado por una estimación robusta, esto es, los outliers reciben un peso menor en la estimación de los coeficientes de la regresión; cf. Fox (2000).
} 
está infrarrepresentado. A medida que se pasa de un perfil a otro, disminuyen los niveles de proporcionalidad y aumentan las ventajas y desventajas de los partidos.

De acuerdo con los gráficos 1 a 5, la circunscripción de Barcelona es la única que presenta un perfil plano de máxima proporcionalidad ${ }^{19}$. En general, todos los partidos con más del 3 por ciento están ligeramente sobrerrepresentados ( $A$ oscila entre 1,00 y 1,05 ) a costa de los pequeños partidos que no superan la barrera legal. Pero todas las tasas de ventaja están sólo ligeramente por encima de 1, puesto que los perdedores tienen poco que perder y los ganadores son demasiados para repartirse el botín. Por su parte, Tarragona y Cataluña en su conjunto presentan un perfil de elevación adelantada. Los partidos resultan penalizados por el sistema electoral cuando obtienen menos del 12-13 por ciento de votos, de modo que las tasas de ventaja llegan hasta el 10 por ciento (esto es, $A=1,10$ ). Estas primas benefician fundamentalmente a CiU y apenas al PSC, sobre todo en Tarragona. Finalmente, Lleida y Girona cuentan con perfiles de proporcionalidad de elevación atrasada. La neutralidad del sistema electoral se alcanza con porcentajes de voto alrededor del 22-23 por ciento. En consecuencia, las tasas de ventaja para los (pocos) partidos que superan este umbral son elevadas: suelen situarse entre $A=1,10$ y $A=1,20$, esto es, entre el 10 y el 20 por ciento.

GRÁFICO 1

Perfil de proporcionalidad en Cataluña

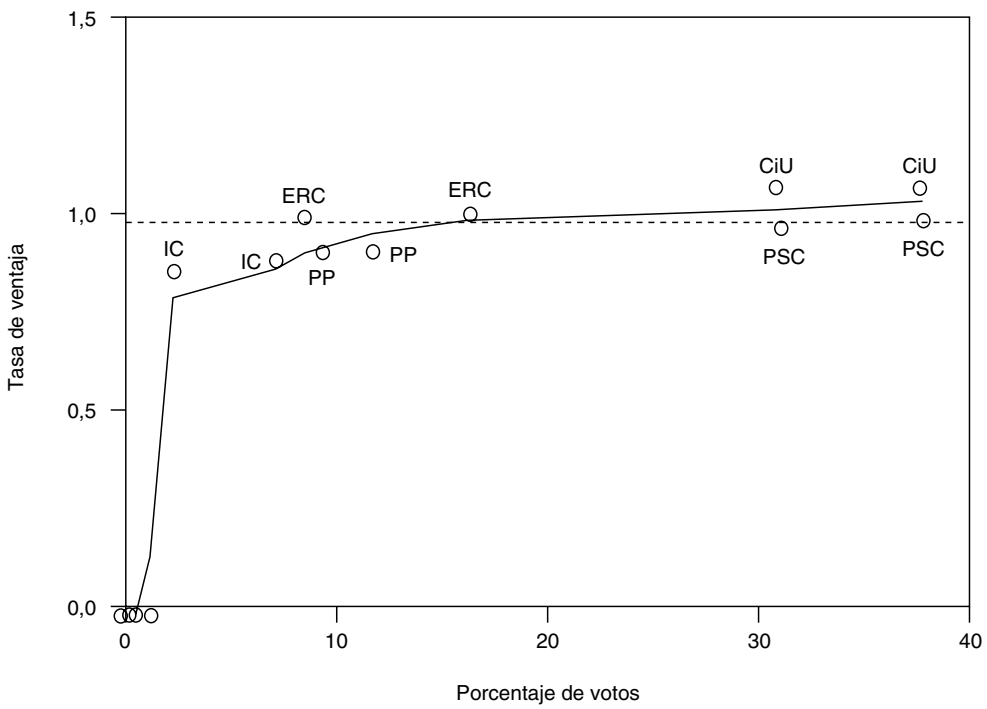

19 En todos los casos se ha optado por un parámetro de curvatura de 0,65 (Fox, 2000). 
GRÁFICO 2

Perfil de proporcionalidad en Lleida

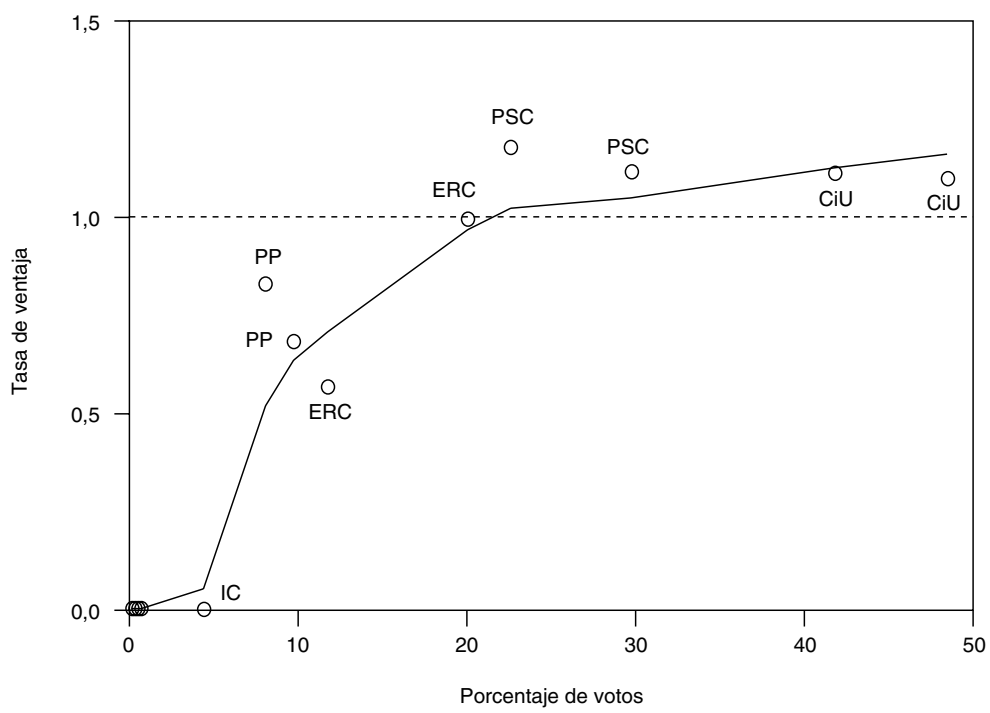

GRÁFICO 3

Perfil de proporcionalidad en Tarragona

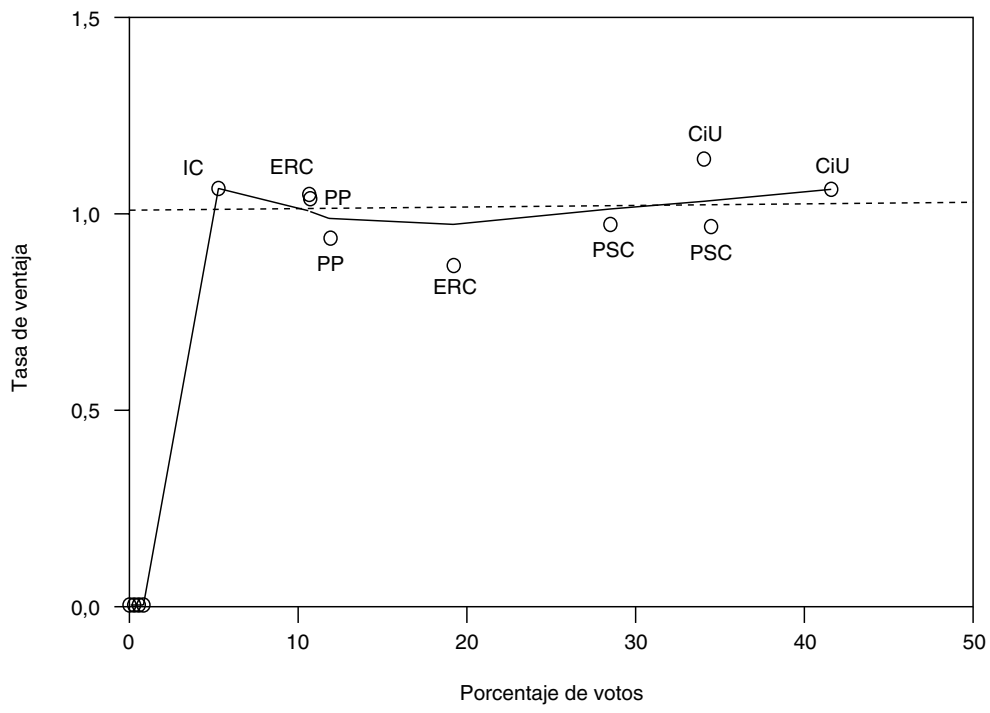




\section{GRÁFICO 4}

Perfil de proporcionalidad en Girona



GRÁFICO 5

Perfil de proporcionalidad en Barcelona

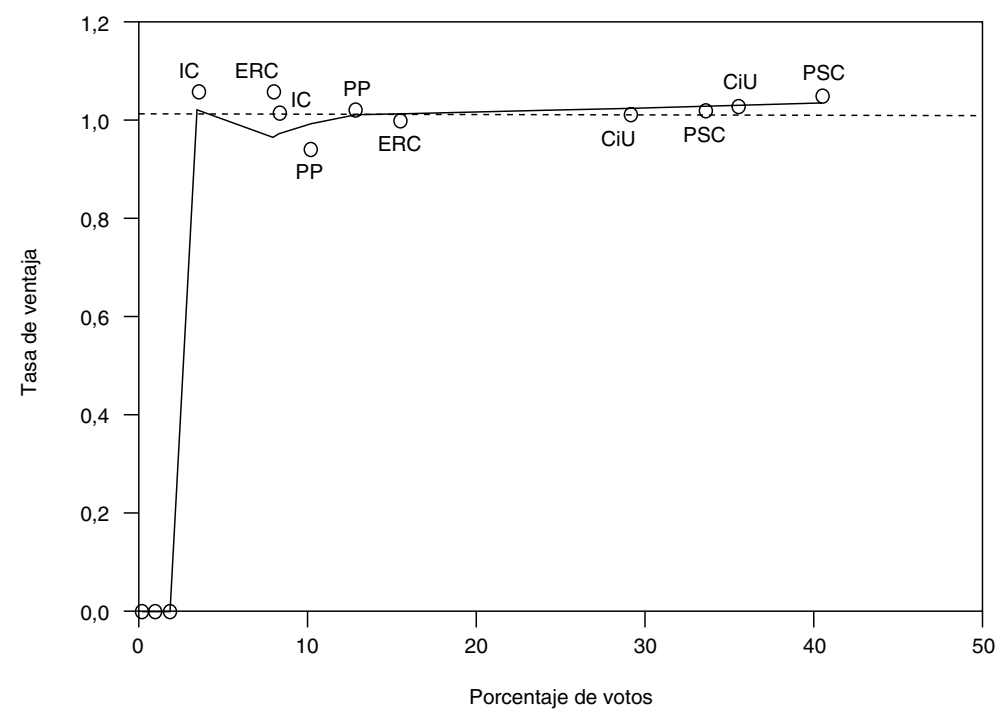




\section{DOS EXPLICACIONES DE MÁS VOTOS Y MENOS ESCAÑOS: PRORRATEO DESVIADO Y EFECTO VARIANZA}

Como se ha observado en los perfiles de proporcionalidad de las cuatro circunscripciones catalanas, la rentabilidad en escaños de las mayorías en votos resulta diferente en cada una de ellas. La relación entre ambas variables es, por supuesto, inversa: a más proporcionalidad, menor sobrerrepresentación. Esta varianza en el premio según la localización del voto pasaría inadvertida si los partidos distribuyeran territorialmente su voto de un modo aleatorio o simétrico, esto es, si no existiera correlación significativa alguna entre la magnitud de las circunscripciones y el voto o, si se prefiere, ninguna tendencia electoral definida en cada provincia. Pero esto no es así. En la medida en que en los distritos de Lleida, Girona y Tarragona, los más rentables electoralmente, son más rurales y contaron en su día con muchos menores niveles de inmigración que Barcelona, partidos de centro-derecha como CiU suelen conseguir la mayoría de los votos y, por tanto, disfrutan de primas mayores que los partidos de centro-izquierda como el PSC, que vencen en Barcelona. Así, en las pasadas elecciones de 2003, las bonificaciones que obtuvo CiU en Tarragona, Lleida y Girona fueron un 759, un 756 y un 341 por ciento mayores que la que alcanzó el PSC en Barcelona (véase infra, tabla 8). La principal consecuencia política de la agregación autonómica de estos dispares niveles de proporcionalidad es que un partido puede conseguir la mayoría de los votos en Girona, Lleida y Tarragona, pero no en el conjunto de Cataluña, y obtener, sin embargo, la mayoría de los escaños. Esto es precisamente lo que ha sucedido en las elecciones autonómicas de 1999 y 2003: pese a recibir menos votos que el PSC, CiU obtuvo más escaños. Si bien la desviación del prorrateo se ha identificado de una manera casi unánime como la responsable de este resultado asimétrico, en las páginas que siguen intentaremos demostrar que tiene que ver fundamentalmente con el llamado «efecto de varianza» de las magnitudes de circunscripción (Monroe y Rose, 2002).

\section{La desviación del prorrateo o malapportionment}

Para la mayoría de los análisis de comentaristas políticos, medios de comunicación y elites partidistas, el desajuste entre votos y escaños sufrido por el PSC y CiU en las últimas elecciones puede explicarse acudiendo al expediente de la desviación del prorrateo. Si las mayorías parlamentarias absolutas de CiU en buena parte de las consultas de los años ochenta y noventa fueron debidas a la manufacturación operada por distintos elementos del sistema electoral a partir de mayorías relativas de votos, ahora el malapportionment ha sido convertido en el responsable de la peculiar manufacturación que permitía obtener una pluralidad de votos y recibir menos escaños que algún partido perdedor. Como lo describía Pallarès (1999: 717), por ejemplo, tras las elecciones de 1999, «debe señalarse que por primera vez la candidatura 
más votada no es la que obtiene mayor número de escaños. En el marco del gran desequilibrio en la distribución territorial de la población de Cataluña (el $75 \%$ vive en la provincia de Barcelona), el sistema electoral vigente (...) prima el valor de representación del voto en las otras circunscripciones y les otorga un mayor número de escaños del que les correspondería proporcionalmente a su población». Los principales medios de comunicación insistieron en variaciones de este argumento. Para La Vanguardia (20 de octubre de 2003: 19), «lo que [sucedió] (...) es que hay provincias sobrerrepresentadas (es decir, que cuentan con más diputados de los que les corresponderían) y otras subrepresentadas (es decir, con menos escaños de los que les tocarían por su peso demográfico)». Y para El País (17 de noviembre de 2003: 18), «la prima en escaños que tienen las provincias de Lleida y Girona, en las que se impuso $\mathrm{CiU}$, sobre la de Barcelona, en la que la primera fuerza fue ampliamente el PSC, dieron a CiU y a Pujol [en 1999] la que sería su última victoria y sus últimos cuatro años de gobierno". Como cabía esperar, la repetición de la situación facilitó la continuidad de las variaciones sobre el malapportionment. Para El País («Qué cambio», 17 de noviembre de 2003: 12), «una ley electoral injusta, que prima el voto en las circunscripciones menores, y una caída del voto socialista superior a la prevista han arrojado uno de los resultados electorales más difícil de imaginar y aún más de gestionar». El principal dirigente del PSC, Pasqual Maragall, atribuyó directamente (en La Vanguardia, 17 de noviembre de 2003: 18) a las disposiciones estatutarias sobre los distritos catalanes su nuevo fiasco electoral. Y Ciutadans pel Canvi (CpC), la plataforma organizativa vinculada con el PSC desde la consulta de 1999, se congratulaba (en www.pelcanvi.org) del triunfo de las candidaturas catalanistas y progresistas «pese a la distorsión de la voluntad popular que supone la ausencia de una Ley Electoral».

La desviación del prorrateo tiene dos efectos opuestos sobre la proporcionalidad electoral. Por un lado, la sobrerrepresentación de las circunscripciones de Girona, Lleida y Tarragona en 5,7 y 5 diputados, respectivamente, posibilita que se reduzcan las desviaciones entre los porcentajes de votos y escaños de los partidos en cada una de ellas: a más escaños a repartir, mejor es el ajuste entre las dos variables. La consecuencia evidente es que las primas a los partidos mayoritarios son ahora menores, puesto que, sobre todo, apenas hay votos sin representación. Pero, por otro lado, cuantos más escaños se reparten en los tres distritos menores, mayor será la influencia relativa de sus resultados en la composición del Parlamento. De esta forma, si bien la desviación del prorrateo aumenta la proporcionalidad electoral local y reduce, por lo tanto, las primas a los partidos mayoritarios, incrementa también la capacidad de distorsión de estas primas, aunque debilitadas, en los resultados electorales agregados para el conjunto de Cataluña. Existe, en fin, una oposición o un trade-off entre la consecución a través del malapportionment de una elevada proporcionalidad en las cuatro circunscripciones que garantiza la representación de todos los partidos significativos y el impedimento de resultados anómalos mediante una mayoría de escaños que no obedece a una similar mayoría de votos. 
La corrección de la desviación del prorrateo, de modo que cada circunscripción disponga de un número de escaños absolutamente proporcional a su peso demográfico, supondría que Barcelona elegiría 102 diputados, Girona 12, Lleida 8 y Tarragona 13: sus cocientes electores/escaño serían, respectivamente, $38.583,38.613,37.785$ y 38.951 . Pero esta reforma electoral dista de ser una panacea. Sus consecuencias negativas son al menos tres. En primer lugar, la gran magnitud de la circunscripción de Barcelona, con nada menos que 102 diputados (que significarían un 76 por ciento del Parlamento catalán), supondría que las elecciones prácticamente se ganan o pierden en ella, puesto que la influencia de los otros tres distritos sería marginal. Al igual que el gerrymandering ${ }^{20}$ resulta tolerable cuando persigue la protección de las minorías o el mantenimiento de tradiciones locales (Mackenzie, 1962: 120122), habría que preguntarse si es admisible que los intereses rurales en Cataluña, presentes fundamentalmente en Girona y Lleida, carezcan de una representación significativa en los resultados que se desprenderían de esta hipotética asignación de escaños (Bosch, 2002). Si Barcelona se lleva la parte del león, también lo hacen los intereses urbanos. En segundo lugar, esta reforma electoral a través de la eliminación del malapportionment no resuelve el problema estructural que desencadena la manufacturación de mayorías parlamentarias. Si la raíz del problema es que en las circunscripciones más rurales, Girona, Lleida y Tarragona, en las que CiU consigue sus mejores resultados, las primas electorales para el partido más votado son muy superiores a las que se alcanzan en Barcelona, esa modificación no corrige esta tendencia, sino que la agrava. Puesto que los escaños en juego en estos tres distritos son ahora menos, la sobrerrepresentación sería más intensa. La desaparición del malapportionment conseguiría simplemente disminuir la influencia de las tres circunscripciones menores en la distribución autonómica de los escaños, pero no la sobrerrepresentación de CiU. Además, una buena parte de la solución quedaría en manos del azar: si en la aplicación de la fórmula D'Hondt el partido mayoritario (CiU) gana sus escaños en estos tres distritos pequeños por un margen estrecho y el segundo (PSC) por uno amplio, al contrario de lo que sucede con la simulación que hemos realizado más adelante (en la tabla 6), de nuevo aumenta la probabilidad de mayorías parlamentarias manufacturadas. Finalmente, y como consecuencia del punto anterior, el riesgo de que un partido con menos votos consiga más escaños sigue existiendo. Es cierto que resulta menos probable, puesto que para que tuviera lugar el margen de votos entre los dos partidos mayoritarios en Barcelona debería ser menor que en la actualidad; pero nada impediría que se repitiera la dinámica de las dos últimas elecciones.

También habría de tenerse en cuenta, de otra parte, la presencia de los efectos «psicológicos» o estratégicos de esta reforma electoral, que se añadirían a los «mecánicos» anteriores. Si las magnitudes de las circunscripciones de Girona, Lleida y Tarragona se redu-

20 El gerrymandering se refiere a una manipulación de la delimitación de los distritos electorales que falsea la representatividad y beneficia intencionadamente a un partido o candidato. 
cen tan significativamente, las elites y los votantes contarían con fuertes incentivos para el comportamiento estratégico. En particular, (1) los partidos que no tienen posibilidades de conseguir algún escaño no competirían o se fusionarían con otros más grandes (así ocurrió, por ejemplo, con la coalición entre el PSC-CpC e IC-V en estos tres distritos en las elecciones de 1999); (2) puesto que las elites concentran sus esfuerzos de movilización en las circunscripciones en las que tienen mejores expectativas, los partidos minoritarios invertirían menores recursos en los distritos más pequeños y, por lo tanto, cosecharían peores resultados; (3) si compiten partidos sin posibilidades de conseguir escaños, surgiría para los electores la posibilidad del voto estratégico y (4) asimismo de la abstención estratégica.

Las consecuencias empíricas de estos probables comportamientos estratégicos añaden aún más incertidumbre a la utilidad de esta reforma electoral. En función de que se pueda rechazar o aceptar total o parcialmente la condición de ceteris paribus en el nuevo escenario de competición respecto al anterior, se agravarían o debilitarían las tendencias desproporcionales implícitas en el cambio institucional, y se avanzaría o retrocedería en la consecución del objetivo perseguido. Y no son pocas las posibilidades. Si, por ejemplo, todo sigue igual salvo que se produzcan niveles significativos de voto estratégico, entonces los partidos mayoritarios podrían conseguir más escaños a costa de los minoritarios y aumentar así sus primas. $\mathrm{Si}$, por el contrario, los partidos (minoritarios) con malas expectativas electorales se retiran como competidores autónomos, podría entonces aumentar la proporcionalidad y reducirse las primas a los partidos mayoritarios. No es posible, pues, realizar predicciones fiables sobre los comportamientos estratégicos de elites y votantes si se produjera la reforma electoral en estos términos, por lo que tampoco cabe tener certeza sobre sus consecuencias inmediatas.

En conclusión, la reforma electoral a través de la desaparición del malapportionment no resuelve definitivamente el problema, sino que lo hace simplemente menos probable. Como mantenemos en el próximo apartado, el problema no radica tanto en el malapportionment por sí mismo, sino sobre todo en el «efecto de varianza» de las magnitudes de los distritos catalanes. Por lo demás, la lógica de una reforma electoral centrada exclusivamente en la absoluta proporcionalidad en la asignación territorial de los escaños conduce a otros efectos no menos sobresalientes y difícilmente aceptables desde distintos criterios normativos o posiciones políticas. Entre ellos cabría citar el incremento de las desviaciones entre los porcentajes de votos y de escaños en las tres circunscripciones menores para reducir las desviaciones de los dos partidos mayoritarios en el conjunto de Cataluña; o la imposibilidad de facto de que partidos minoritarios, aunque relevantes, como IC-V, puedan conseguir escaños fuera de Barcelona; o, en fin, la desaparición virtual de la representación de los intereses rurales del Parlamento catalán. 


\section{El efecto de varianza de las magnitudes de circunscripción}

Frente a este consenso sobre la importancia decisiva del malapportionment en la composición del Parlamento autonómico, nosotros creemos que la posibilidad de que un partido con menos votos que otro en el conjunto de Cataluña pueda conseguir más escaños se desprende del llamado «efecto de varianza» de las magnitudes de distrito. Como escriben Monroe y Rose (2002: 68; cursivas en el original), sus más recientes estudiosos, «el punto de partida (...) radica en que el bien conocido efecto de la magnitud de distrito es un efecto del primer momento, por así decir, de la distribución de la magnitud (...). El efecto de varianza (...) [recoge] los efectos del segundo momento de la magnitud de distrito. Mientras que los efectos son de "segundo orden" en un sentido técnico, son en cierto sentido más importantes que los (...) del primer momento. Éstos (...) conciernen a cuántos jugadores se sientan en la mesa política. El efecto de varianza ayuda a determinar qué jugadores están en las mesas y qué cartas tienen. Esto es, la variación de la magnitud de distrito tiene efectos (...) partidistas predecibles». Dicho de otro modo, en los sistemas electorales que cuentan con circunscripciones de distintas magnitudes, la magnitud de distrito tiene dos efectos complementarios. En primer lugar, al menos desde el análisis empírico pionero de Rae (1971), es bien conocido que los sistemas de partidos dependen en gran medida de esta variable. Ceteris paribus, el número de partidos o la fragmentación del sistema de partidos aumenta, aunque no linealmente, a medida que se incrementan los escaños en juego. Como Cox (1997) ha demostrado, el número máximo de partidos o candidatos viables en todos los sistemas electorales es igual a la magnitud de distrito más uno; se trata de la denominada regla $M+1$. Por tanto, a lo largo de un sistema electoral con distintas magnitudes de distrito el número de competidores varía. Pero, en segundo lugar, cuando alguna característica política destacada del electorado que influye en las preferencias electorales se distribuye a lo largo de las circunscripciones de tal modo que está correlacionada con la magnitud de distrito, entonces la variación en las magnitudes de distrito tiene también efectos políticos partidistas. Y esto es precisamente lo que sucede tanto en las elecciones autonómicas en Cataluña como en las generales en Espa$\tilde{n} a^{21}$. Los distritos coinciden con las provincias, y sus magnitudes guardan cierta proporcionalidad, aunque no sea perfecta, con la población. La varianza resultante en la magnitud de los distritos es acusada: entre 15 y 85 escaños en Cataluña, y entre 1 y 33 en España. En la medida en que las áreas más pobladas son también las que tienen mayores densidades de

\footnotetext{
21 Para el caso español, algunas propuestas han bordeado el concepto central del efecto de varianza, aunque no han llegado a plantearlo de forma sistemática. Así, Santamaría (1996a: 41 y 1996b: 241) ha llamado la atención sobre la mucha mayor importancia alcanzada por los sesgos mayoritarios del sistema para el Congreso de los Diputados cuando el primer partido es conservador, como la Unión de Centro Democrático (UCD) o el PP; Penadés (1999: 314-327) ha analizado el «premio a la localización» que ha permitido a los mayores partidos, pero sobre todo a UCD y al PP, aprovecharse de los varios mecanismos de ventajas acumulados por la heterogeneidad interna del sistema electoral español; y Capo (1999: 64-66) ha examinado un "coeficiente de rendimiento electoral" para dar cuenta de las distorsiones del sistema electoral que combinan efectos propios de la magnitud de las circunscripciones o de la fórmula electoral con otros derivados de la distribución territorial de la fuerza de los partidos.
} 
población, las zonas urbanas están contenidas en los distritos de magnitud elevada, y las rurales en los de magnitud baja. Puesto que la competición electoral depende fundamentalmente del número de escaños en juego, un partido mayoritario que alcanza sus mejores resultados en las áreas urbanas (como el PSC) tiene que competir con más rivales (y más fuertes) que otro que lo hace en las rurales (como CiU). De este modo, las preferencias partidistas en los distritos rurales se convierten más fácilmente en representación parlamentaria efectiva que las de los urbanos. El efecto de varianza de las magnitudes de distrito tiene, en este sentido, tres consecuencias partidistas (Monroe y Rose, 2002: 69):

i) Ceteris paribus, los intereses urbanos tienen más dificultades que los rurales para traducir el apoyo electoral latente en una representación parlamentaria no fragmentada. Puesto que a medida que aumenta la magnitud de una circunscripción también lo hace el número de competidores, los partidos que representan en mayor medida los intereses urbanos (como el PSC) tendrán más rivales intrabloque que los partidos que más representan los intereses rurales (como CiU). En consecuencia, la fragmentación electoral y parlamentaria de los partidos urbanos será mayor que la de los rurales.

ii) Ceteris paribus, los intereses urbanos tienen más dificultades que los rurales para traducir el apoyo electoral latente en una representación parlamentaria total. Como corolario del punto anterior, el partido o los partidos mayoritarios que representan en mayor medida los intereses urbanos (como el PSC) serán menos fuertes en sus circunscripciones más propicias, las grandes, que el partido o los partidos mayoritarios que más representan los intereses rurales (como $\mathrm{CiU}$ ) en las suyas, las pequeñas. Por lo tanto, los primeros tenderán a conseguir menos escaños.

iii) Y, ceteris paribus, los partidos urbanos mayoritarios tienen más dificultades que los rurales para traducir el apoyo electoral latente en representación parlamentaria. La combinación de los anteriores mecanismos significa que los partidos (y los votantes) que representan en mayor medida los intereses rurales (como CiU) tienen más incentivos para la coordinación, o menos para la descoordinación, y pueden de este modo obtener más escaños.

En este efecto de varianza se combinan, pues, relaciones mecánicas y psicológicas. En un caso, la mayor desproporcionalidad electoral en los distritos con zonas rurales predominantes o/y con magnitudes más bajas acentúa las primas electorales de los dos partidos mayoritarios, sobre todo del primero. Dicho de modo distinto, es más rentable en términos de escaños ser el partido más votado en un distrito de magnitud baja que en otro de alta; las primas electorales recogidas en la tabla 8 lo confirman plenamente. En el otro aparecen fuertes incentivos para el comportamiento estratégico de elites y votantes en los distritos más pequeños; sus principales modalidades han sido ya descritas con anterioridad. 
En síntesis, el argumento del efecto de varianza señala que cuando existe una correlación entre la magnitud de los distritos y alguna característica política destacada del electorado, los escaños se distribuyen de manera diferente con un sistema electoral que tiene una amplia varianza en la magnitud de las circunscripciones que con otro que no la tuviera. Y no se trata de un mecanismo menor. De acuerdo con los cálculos de Monroe y Rose (2002: 85-86) para 24 elecciones celebradas en 16 países desde los años setenta, el efecto de varianza es responsable de la redistribución de un 17 por ciento de la representación parlamentaria. Las primas alcanzadas por los principales partidos españoles son también significativas, especialmente si se trata de UCD y el PP (Penadés, 1999: 321-322; Capo, 1999: 68-70). Para ilustrar su lógica y consecuencias en el caso catalán, imaginemos, con los datos de las elecciones autonómicas de 2003, que la circunscripción de Barcelona, que cuenta con 85 escaños, se dividiera en otros tantos distritos uninominales, mientras que Girona, Lleida y Tarragona no cambiaran. Supongamos, además, que cada uno de estos 85 distritos fuera un microcosmos de la provincia, de modo que en todos ellos el PSC-CpC sea el partido más votado, con el 33,18 por ciento de los votos. En lugar de conseguir los 29 diputados cuando la circunscripción es plurinominal, los socialistas habrían obtenido los 85 en juego. En otras palabras, el PSC pasaría de una sobrerrepresentación de 0,63 puntos porcentuales a otra de 66,51. La agregación de los resultados de los cuatro distritos supondría que el PSC-CpC alcanzaría 102 diputados, mientras que CiU habría de conformarse con sólo 21.

Si la manufacturación de mayorías parlamentarias en Cataluña depende ante todo de la extraordinaria variabilidad en las magnitudes de los cuatro distritos, y marginalmente de la desviación del prorrateo, las soluciones pasarían por limitar ese efecto de varianza. En principio, las posibilidades de la ingeniería electoral se basarían en dos criterios: aumentar el umbral efectivo de la circunscripción de Barcelona o reducir el de las de Girona, Lleida y Tarragona. El primer criterio supondría: i) desagregar la provincia de Barcelona en varios distritos de menor magnitud, o ii) emplear barreras legales provinciales más elevadas o establecer una barrera autonómica. Por su parte, el segundo criterio implicaría: a) combinar las provincias de Girona, Lleida y Tarragona en menos distritos y de mayor magnitud; b) incrementar todas las magnitudes de distrito; c) añadir un segundo nivel en el que se sumen los votos para repartir escaños autonómicos suplementarios; o d) crear un distrito único 22 .

Naturalmente, los costes e incertidumbres de estos dos grupos de reformas son variables. Sus consecuencias políticas son, sin embargo, muy distintas. Las primeras persiguirían acentuar la desproporcionalidad y las primas en la circunscripción de Barcelona y, por lo tanto, facilitarían la formación de mayorías parlamentarias más amplias y gobiernos más fuertes u homogéneos en comparación con el escenario actual. Además, la multiplicación de distri-

\footnotetext{
22 La corrección del malapportionment supone precisamente avanzar en la dirección del distrito único.
} 
tos podría suponer una mayor cercanía entre políticos y votantes, así como un mayor control de las acciones del agente. Pero esta ingeniería electoral se topa con dificultades importantes. ¿Con qué criterios se fijarían las nuevas circunscripciones? ¿Cómo evitar los riesgos de gerrymandering que se han eliminado gracias a los distritos provinciales? ¿Y hasta qué punto son legal y políticamente tolerables las barreras legales superiores al 5 por ciento? Las segundas reformas, por el contrario, pretenderían disminuir la desproporcionalidad en las circunscripciones de Girona, Lleida y Tarragona. La consecuencia esperada no es otra que una mayor fragmentación en el sistema de partidos, de la que se derivaría la mayor dificultad para generar mayorías parlamentarias amplias y la mayor probabilidad de gobiernos minoritarios o de coalición.

\section{SIMULACIONES DE LOS RESULTADOS DE DISTINTAS REFORMAS ELECTORALES}

Para observar el alcance de las explicaciones de este resultado de más votos y menos escaños basadas en la desviación del prorrateo y la varianza de las magnitudes de las circunscripciones, revisaremos su impacto diferenciado en distintas propuestas de reforma electoral. Con algunas de ellas efectuaremos simulaciones de los resultados electorales en función de los habidos en la consulta de $2003^{23}$. Como resulta obligado aclarar, la interpretación de dichos resultados exige una prudencia elemental, puesto que asumen el supuesto de ceteris paribus derivado de la imposibilidad de que en cada uno de los distintos escenarios suministrados por cada sistema electoral el comportamiento de partidos y votantes resulte similar al registrado en noviembre de 2003. Sea como fuere, un problema adicional consiste en determinar qué sistema electoral se selecciona y qué variantes se utilizan en la correspondiente simulación. Dado las más de dos décadas transcurridas sin ley electoral propia, se han formulado numerosas propuestas por los partidos (sobre todo, en sus respectivos programas para las elecciones autonómicas), por observadores políticos y por distintas comunidades de expertos. La práctica totalidad de esas propuestas coincide en subrayar la igualdad del voto también por criterios territoriales, la proporcionalidad entre votos y escaños a través de una modificación de la fórmula o de las circunscripciones electorales y el mantenimiento de una barrera electoral de alcance intermedio (cf., por ejemplo, Vallès 2002). Y muchos de ellos convergen además en alguna variante del sistema electoral alemán (cf., también por ejemplo, Bosch, 1999 y 2002). En enero de 2003, el Grupo de Socialistas-Ciutadans pel Canvi en el Parlamento catalán presentó una proposición de Ley Electoral de Cataluña que pretendía explícitamente la adaptación del sistema alemán de representación proporcional personalizada. Sus principales elementos incluían las 41 comarcas como circunscripciones uninominales (a las que se añadía otra para catalanes residen-

23 Para otras simulaciones, realizadas con los resultados de elecciones anteriores y numerosas variantes de sistemas electorales, pueden verse Vallès (1982), Bosch (1999), Capo (2002), Castro (2002) o Magre y Pont (2002). 
tes en el extranjero) para elegir por mayoría relativa a otros tantos candidatos, la autonómica como circunscripción única para completar la asignación de un total de 135 escaños mediante la fórmula D'Hondt entre las listas «nacionales» de los partidos y una barrera electoral del 3 por ciento ${ }^{24}$. Durante la campaña electoral de noviembre de 2003, el PSC-CpC mantuvo naturalmente esta propuesta como uno de sus criterios fundamentales para relanzar la «calidad democrática» del autogobierno en Cataluña, mientras que ERC incluía en su programa una ley electoral propia como medida para «profundizar» la democracia, e ICVEUiA proponía que la futura ley electoral mejore la proporcionalidad territorial y política de los votos de los ciudadanos y permita el voto preferencial a candidatos específicos ${ }^{25}$. En fin, el acuerdo suscrito por PSC-CpC, ERC e ICV-EUiA para la formación del gobierno catalán en diciembre de 2003 recogía distintos criterios para una futura Ley Electoral de Cataluña, entre los que se encontraban los de la igualdad de voto de los electores, la proporcionalidad entre votos y escaños, la expresión de la diversidad territorial catalana y una representación equitativa en materia de género. Aunque en términos relativamente imprecisos, se preveía además una aplicación del modelo alemán mediante las circunscripciones uninominales de ámbitos territoriales por el momento indeterminados y una circunscripción única para completar un total indeterminado de escaños a repartir entre las listas «nacionales» de los partidos mediante la fórmula electoral D'Hondt y una barrera electoral del 3 por ciento en el ámbito catalán ${ }^{26}$.

Dados estos antecedentes, limitaremos la realización de simulaciones a sólo tres propuestas de reforma electoral. Serán las siguientes:

1. ${ }^{\text {a }} \quad$ La desaparición de malapportionment mediante la ya comentada distribución de los escaños entre los distritos de un modo totalmente proporcional a la población de cada uno de ellos; los restantes elementos del sistema electoral vigente no cambiarían. Barcelona pasaría a elegir así 102 diputados; Girona, 12; Lleida, 8, y Tarragona, 13.

2. ${ }^{\text {a }}$ El establecimiento de un distrito único; los restantes elementos del sistema electoral vigente tampoco se modificarían.

\footnotetext{
24 La proposición está recogida de www.psc.es.

25 Los programas de ERC y de ICV-EUiA se encuentran en www.esquerra.org y www.ic-v.org, respectivamente.

26 Estos criterios se encuentran recogidos en el Acord per a un Govern catalanista i d'esquerres a la Generalitat de Catalunya, de 14 de diciembre de 2003, que está incluido en www.psc.es. Cabe señalar que, durante las negociaciones entre CiU y ERC para la formación de un gobierno entre ambos, CiU se comprometió a consensuar una nueva ley electoral«que se base en la proximidad con el ciudadano y en la representatividad de todos los territorios», y que preveía «la elección de un diputado por comarca y distrito de Barcelona, y elección proporcional del resto de la lista a través de las circunscripciones correspondientes a las nueve regiones o veguerías, excepto tres diputados que serían escogidos por catalanes residentes fuera de Cataluña»; en Document marc per un Govern de coalició CiU-ERC, de 27 de noviembre de 2003, incluido en www.convergencia-i-unio.org.
} 
3. ${ }^{\text {a }} \quad$ Y la adopción de un sistema electoral de miembros-mixtos, según la denominación de Shugart y Wattenberg (2001b). Se trata de la propuesta de reforma electoral que se defiende en el Pacto de gobierno entre el PSC-CpC, ERC e ICV-EUiA. Dada su imprecisión en algunos aspectos fundamentales (como el número y naturaleza de los distritos uninominales o el tamaño del Parlamento), hemos completado el contenido del Pacto con la proposición de Ley Electoral presentada en enero de 2003 por el PSC-CpC. De este modo, y al igual que en Alemania, los 135 diputados se elegirían en dos niveles de circunscripción y con fórmulas electorales distintas. En primer lugar, 41 diputados se elegirían en otros tantos distritos uninominales basados en las comarcas y de acuerdo con una fórmula de mayoría relativa. En segundo lugar, una circunscripción única autonómica determinaría cuántos escaños le corresponderían a cada partido según la fórmula D’Hondt y una barrera legal del 3 por ciento de los votos válidos. Cada candidatura conseguiría estrictamente el número de escaños que se contemplan en la circunscripción autonómica, de manera que los distritos uninominales sólo tratan de propiciar una mayor proximidad de los representantes a los representados. Los efectos de este sistema de miembros-mixtos son, en fin, idénticos a los del distrito único.

En la tabla 6 se presenta la composición del Parlamento catalán que resultaría de la utilización de alguna de esas tres variantes del sistema electoral. Ceteris paribus, tanto la corrección del prorrateo como el distrito único y el sistema de miembros-mixtos hubieran evitado el resultado de más votos y menos escaños: en los tres escenarios institucionales el PSC y CiU empatarían a escaños. Pero si en los dos últimos casos este resultado se consigue a través de un aumento significativo de la proporcionalidad, en el supuesto de la desaparición del malapportionment la proporcionalidad electoral disminuiría, sobre todo en las circunscripciones menores. En efecto, de acuerdo con el índice de Rose, la proporcionalidad autonómica aumenta en cerca de 2 puntos porcentuales en los sistemas electorales de miembros-mixtos y distrito único frente al vigente, y disminuye en casi medio punto porcentual cuando se elimina el malapportionment (tabla 7). En este supuesto, la proporcionalidad de Barcelona apenas experimentaría cambios, pero las de Girona, Lleida y Tarragona caerían en 3, 7 y 5 puntos porcentuales, respectivamente. Es decir, para que el sistema electoral sea más justo en la traducción de los votos del PSC y CiU en escaños, debe hacerse más injusto para los restantes partidos, especialmente cuanto más minoritarios sean.

Finalmente, y en relación con nuestro principal argumento, debemos destacar que si bien los sistemas electorales de distrito único y de miembros-mixtos garantizarían que nunca se produjeran resultados de más votos y menos escaños, no puede decirse lo mismo cuando la reforma comprenda exclusivamente la corrección del prorrateo desviado. El empate en escaños entre el PSC y CiU en este caso tiene mucho que ver con el azar: mientras que CiU gana su último escaño en cada circunscripción por un amplio margen, el PSC lo hace por uno es- 
trecho. Si se invirtiese esta pauta, y nada impide que sea así en otras ocasiones, de nuevo se generaría una mayoría parlamentaria artificial. Además, los efectos esperados de los comportamientos estratégicos de las elites partidistas y de los votantes que estas reformas desencadenarían son muy distintos. En los sistemas electorales de distrito único y de miembros-mixtos, no contarían por definición en la consecución del objetivo esperado. Cuando solamente se corrige el malapportionment, los efectos psicológicos son, sin embargo, opuestos a los mecánicos: si se reduce la magnitud de las circunscripciones de Girona, Lleida y Tarragona, se incentivaría la coordinación electoral a través de la retirada de competidores, la formación de coaliciones o el voto estratégico, por ejemplo, y, en consecuencia, se incrementarían las primas a los partidos mayoritarios (tabla 8). De este modo, la influencia de los resultados en estos tres distritos en la composición del Parlamento se vería nuevamente incrementada.

TABLA 6

Composición del Parlamento catalán según distintas variantes del sistema electoral con los resultados de noviembre de 2003

\begin{tabular}{lrrrr} 
& & & Sistema electoral \\
\cline { 2 - 5 } Partidos & Vigente & Sin malapportionment & Distrito único & Miembros-mixtos \\
\hline PSC-CpC & 42 & 44 & 43 & 43 \\
CiU & 46 & 44 & 43 & 43 \\
ERC & 23 & 24 & 23 & 23 \\
PP & 15 & 15 & 16 & 16 \\
ICV-EUiA & 9 & 8 & 10 & 10 \\
\hline Total & 135 & 135 & 135 & 135
\end{tabular}

FUENTE:

Elaboración propia 
TABLA 7

Índices de proporcionalidad de Rose según distintas variantes del sistema electoral con los resultados de noviembre de 2003

Sistema electoral

\begin{tabular}{|c|c|c|c|c|}
\hline Circunscripciones & Vigente & Sin malapportionment & Distrito único & Miembros-mixtos \\
\hline Barcelona & 98,58 & 98,32 & & \\
\hline Girona & 95,95 & 93,12 & & \\
\hline Lleida & 91,23 & 84,68 & & \\
\hline Tarragona & 94,89 & 89,54 & & \\
\hline Cataluña & 96,73 & 96,33 & 98,52 & 98,52 \\
\hline
\end{tabular}

FUENTE:

Elaboración propia.

\section{TABLA 8}

Primas electorales del PSC-CpC y de CiU según distintas variantes del sistema electoral con los resultados de noviembre de 2003

Sistema electoral

\begin{tabular}{|c|c|c|c|c|c|}
\hline \multirow{2}{*}{\multicolumn{2}{|c|}{ Circunscripciones y partidos }} & \multirow{3}{*}{$\begin{array}{r}\text { Vigente } \\
0,63\end{array}$} & \multirow{3}{*}{$\begin{array}{r}\text { Sin malapportionment } \\
0,82\end{array}$} & \multirow{3}{*}{ Distrito único } & \\
\hline & & & & & Miembros-mixtos \\
\hline \multirow[t]{2}{*}{ Barcelona } & PSC & & & & \\
\hline & $\mathrm{CiU}$ & 0,40 & 0,40 & & \\
\hline \multirow[t]{2}{*}{ Girona } & PSC & $-0,31$ & 1,16 & & \\
\hline & $\mathrm{CiU}$ & 2,15 & 2,64 & & \\
\hline \multirow[t]{2}{*}{ Lleida } & PSC & 4,00 & 2,34 & & \\
\hline & $\mathrm{CiU}$ & 4,76 & 8,09 & & \\
\hline \multirow[t]{2}{*}{ Tarragona } & PSC & $-0,73$ & 2,26 & & \\
\hline & CiU & 4,78 & 4,38 & & \\
\hline \multirow[t]{2}{*}{ Cataluña } & PSC & $-0,34$ & 1,13 & 0,39 & 0,39 \\
\hline & CiU & 2,86 & 1,38 & 0,64 & 0,64 \\
\hline
\end{tabular}

\section{NOTA:}

En cada circunscripción, se han subrayado con cursiva los partidos con las mayores primas electorales.

FUENTE:

Elaboración propia. 


\section{CONCLUSIONES}

En este artículo hemos demostrado que la manufacturación de mayorías parlamentarias en las dos últimas elecciones autonómicas catalanas no ha sido debida tanto a la desviación del prorrateo, como suele señalarse por dirigentes políticos y expertos académicos, como a la notable variabilidad de las magnitudes de las cuatro circunscripciones. Las reformas electorales basadas en la corrección del malapportionment no garantizarían, por lo tanto, que no se repita ese resultado, sino que simplemente reducirían su probabilidad. Para asegurar que el partido más votado sea también el que siempre disponga de más escaños, las posibilidades de la ingeniería electoral pasan por limitar el efecto de varianza a través de la convergencia entre las magnitudes o el umbral efectivo de la circunscripción de Barcelona y las de Girona, Lleida y Tarragona. Las alternativas son múltiples y entrañan costes y dificultades variables. Por nuestra parte, nos hemos limitado a realizar unas simulaciones elementales con dos reformas básicas del sistema electoral vigente en las elecciones autonómicas (la proporcionalidad en el prorrateo de escaños para cada circunscripción y la implantación de un distrito único), a las que hemos añadido el supuesto más complejo de un nuevo sistema electoral basado, de un modo u otro, en el modelo alemán. Los resultados han reforzado la importancia del efecto de varianza de las circunscripciones catalanas, y han relativizado la relevancia que suele atribuírsele, sin embargo, al malapportionment.

Pero no podemos por menos que finalizar recordando, una vez más, la obviedad de que la reforma de un sistema electoral es una decisión política, adoptada por políticos y en función de intereses políticos (Montero, 1997: 10). Como demuestra la extraordinaria estabilidad de los sistemas electorales de los países europeos en el último siglo, las reformas electorales son de ocurrencia tan excepcional como resultan onerosas para los actores partidistas implicados en ellas, que han solido limitarse a la modificación de elementos secundarios. En Cataluña, la ausencia de una ley electoral propia es, cuando menos, un indicador ilustrativo de la existencia de serios desacuerdos entre las elites políticas sobre sus dimensiones fundamentales. Es evidente que, tras más de dos décadas de autogobierno con sólo muestras ocasionales y siempre abandonadas de elaborar una ley electoral propia, esos desacuerdos han sido también duraderos. El predominio de CiU desde 1980, atrapada entre demandas contradictorias para su estrategia política y renuente en cualquier caso a suscribir el necesario acuerdo con las restantes fuerzas políticas, ha contribuido asimismo a tan anómalo resultado institucional (Capo, 2002). En diciembre de 2003, el nuevo gobierno de coalición ha conferido la máxima importancia a la elaboración de una ley electoral autonómica ${ }^{27}$. Parece

\footnotetext{
27 En el documento ya citado del Acord per a un Govern catalanista i d'esquerres, la propuesta de elaboración del «proyecto de ley sobre elecciones al Parlamento de Cataluña» aparece recogida en un lugar prioritario, y dentro de la sección dedicada al «reforzamiento de la calidad democrática del sistema político», dirigido, junto con otras medidas, a corregir "las causas que provocan el alejamiento ciudadano de las instituciones y la desconfianza respecto a sus decisiones».
} 
un momento particularmente propicio para continuar el debate sobre los diagnósticos de los problemas que pretenden solventarse, sus posibles alternativas y sus probables consecuencias. Esta nota quiere precisamente contribuir a ese debate a través de la presentación crítica de los mecanismos y de los efectos causales que entran en juego en las múltiples interacciones entre el sistema electoral y el sistema de partidos en Cataluña.

\section{REFERENCIAS}

BOSCH, Agustí (1999): «Els sistema electoral. Una proposta per al Parlament de Catalunya», en Joan Font y A. Bosch, Participació i sistema electoral. Propostes per al debat, Barcelona: Editorial Mediterrània.

- (2002): «Una proposta de sistema electoral», en Reflexions al voltant de le Llei electoral catalana, Barcelona: Fundació Carles Pi i Sunyer d'Estudis Autonòmics i Locals.

CAPO, Jordi (1999): «Sistema electoral y gobernabilidad española», Revista Española de Ciencia Política, 1: 55-80.

- (2002): "Crítica i proposta per a un sistema electoral per Catalunya», en Reflexions al voltant de le Llei electoral catalana, Barcelona: Fundació Carles Pi i Sunyer d'Estudis Autonòmics i Locals.

CASTRO, Carles (2002): «Reflexions i escenaris pel disseny d'un model electoral per Catalunya», en Reflexions al voltant de le Llei electoral catalana, Barcelona: Fundació Carles Pi i Sunyer d’Estudis Autonòmics i Locals.

COLOMÉ, Gabriel (1998): “Quince años de experiencia electoral autonómica: el caso de Cataluña», en Juan Montabes (ed.), El sistema electoral a debate. Veinte años de rendimientos del sistema electoral español (1977-1997), Madrid: Centro de Investigaciones Sociológicas/Parlamento de Andalucía.

COLOMER, Josep M. (2001): Instituciones políticas, Barcelona: Ariel.

COX, Gary W. (1997): Making votes count. Strategic coordination in the world's electoral systems, Cambridge: Cambridge University Press.

FOX, John (2000): Multiple and generalized nonparametric regression, Beverly Hills: Sage Publications.

GALLAGHER, Michael (1991): «Proportionality, disproportionality and electoral systems», Electoral Studies, 10 : 33-51.

LAAKSO, Markku, y TAAGEPERA, Rein (1979): «Effective number of parties. A measure with applications to West Europe», Comparative Political Studies, 12: 3-27.

LAGO, Ignacio (2003): El voto estratégico en las elecciones generales en España (1977-2000): efectos y mecanismos causales en la explicación del comportamiento electoral, Madrid: Tesis doctoral, Instituto Juan March.

LIJPHART, Arend (1995): Sistemas electorales y sistemas de partidos. Un estudio de veintisiete democracias, 19451990, Madrid: Centro de Estudios Constitucionales.

LINZ, Juan J.; MONTERO, José Ramón, y RUIZ, Antonia M. (2004): «Elecciones y política», de próxima publicación en Albert Carreras y Xavier Tafunell (eds.), Estadísticas históricas de España, 1800-2000, Madrid: Fundación BBVA.

LOOSEMORE, John, y HANBY, Victor J. (1979): «The theoretical limits of maximum distortion: some analytic expressions for electoral systems», British Journal of Political Science, 1: 467-477.

MACKENZIE, W. J. M. (1962): Elecciones libres, Madrid: Tecnos.

MACKIE, Thomas T., y ROSE, Richard (1991): The international almanac of electoral history, Londres: Macmillan, $3 .^{\mathrm{a}}$ ed. 
MAGRE, Jaume, y PONT, Àngels (2002): «Una proposta de sistema electoral per a Catalunya», en Reflexions al voltant de le Llei electoral catalana, Barcelona: Fundació Carles Pi i Sunyer d’Estudis Autonòmics i Locals.

MONROE, Burt L. (1994): «Disproportionality and malapportionment: measuring electoral inequality», Electoral Studies, 13: $132-149$.

MONROE, Burt L., y ROSE, Amanda G. (2002): «Electoral systems and unimagined consequences. Partisan effects of districted proportional representation», American Journal of Political Science, 46: 67-89.

MONTERO, José Ramón (1997): «El debate sobre el sistema electoral: rendimientos, criterios y propuestas de reforma», Revista de Estudios Políticos, 95: 9-46.

MONTERO, José Ramón; LLERA, Francisco J., y TORCAL, Mariano (1992): «Sistemas electorales en España: una recapitulación», Revista Española de Investigaciones Sociológicas, 58: 7-56.

PALLARÈS, Francesc (1998): «Los sistemas electorales en las Comunidades Autónomas: aspectos institucionales», en Juan Montabes (ed.), El sistema electoral a debate. Veinte años de rendimientos del sistema electoral español (19771997), Madrid: Centro de Investigaciones Sociológicas/Parlamento de Andalucía.

- (1999): «Las elecciones autonómicas del 19 de octubre de 1999 en Cataluña», en Joaquín Tornos (ed.), Informe Comunidades Autónomas 1999, Barcelona: Instituto de Derecho Público.

PENADÉS, Alberto (1999): «El sistema electoral español (1977-1996)», en Juan Luis Paniagua y Juan Carlos Monedero (eds.), En torno a la democracia en España. Temas abiertos del sistema político español, Madrid: Tecnos.

- (2000): Los sistemas elementales de representación, Madrid: Tesis doctoral, Instituto Juan March.

RAE, Douglas W. (1971): The political consequences of electoral laws, New Haven: Yale University Press, $2 .^{a}$ ed.

ROSE, Richard (1984): «Electoral systems: a question of degree or of principle?», en Arend Lijphart y Bernard Grofman (eds.), Choosing an electoral system: issues and alternatives, Nueva York: Praeger.

SAMUELS, David, y SNYDER, Richard (2001): «The value of a vote: malapportionemnt in comparative perspective», British Journal of Political Science, 31: 651-671.

SANTAMARÍA, Julián (1996a): «El sistema electoral español, entre el pasado y el futuro», Temas para el Debate, 18: 40-45.

- (1996b): «El debate sobre las listas electorales», en Antonio Porras (ed.), El debate sobre la crisis de la representación política, Madrid: Tecnos.

SHUGART, Mattew Soberg, y WATTENBERG, Martin P. (2001a): «Conclusion: are mixed-member electoral systems the best of both worlds?», en M. S. Shugart y M. P. Wattenberg (eds.), Mixed-member electoral systems: the best of two worlds?, Oxford: Oxford University Press.

- (2001b): «Mixed-member electoral systems: a definition and typology», en M. S. Shugart y M. P. Wattenberg (eds.), Mixed-member electoral systems: the best of two worlds?, Oxford: Oxford University Press.

TAAGEPERA, Rein, y SHUGART, Mathew S. (1989): Seats and votes: the effects and determinants of electoral systems, New Haven: Yale University Press.

TAAGEPERA, Rein, y GROFMAN, Bernard (2003): «Mapping the indices of seats-votes disproportionality and interelection volatility", Party Politics, 6: 659-677.

VALLÈS, Josep Maria (1982): Reforma electoral i coordenades polítiques, Barcelona: Fundació Jaume Bofill/Edicions de la Magrana. 
VALLÈS, Josep Maria (1990): «Elección de los diputados», en Instituto de Estudios Autonómicos, Comentarios sobre el Estatuto de Autonomía de Cataluña, Barcelona: Institut d'Estudis Autonòmics.

- (2002): «Els criteris básicos de la futura llei electoral catalana», en Reflexions al voltant de le Llei electoral catalana, Barcelona: Fundació Carles Pi i Sunyer d’Estudis Autonòmics i Locals.

\section{ABSTRACT}

In this paper we review (1) the electoral mechanisms that explain the victories in terms of parliamentary seats but not in terms of votes, achieved by the CiU party in the last two Catalan autonomous regional elections and (2) the possible electoral reforms that may be undertaken. Our main conclusion is that artificial $\mathrm{CiU}$ parliamentary majorities are not so much related to pro rata deviation as they are to variations of district magnitudes or «the variance effect».

Key words: Electoral Systems, Catalonia, Pro Rata Deviation, Variance Effect, Parliamentary Seats, Votes. 